\title{
Resident Handoffs: Appreciating Them as a Critical Competency
}

\author{
Jennifer S. Myers, $M D^{7}$ and Lisa M. Bellini, $M D^{2}$ \\ ${ }^{1}$ Associate Professor of Clinical Medicine, Patient Safety Officer, Hospital of the University of Pennsylvania, Director of Quality and Safety \\ Education, Perelman School of Medicine, Philadelphia, USA; ${ }^{2}$ Department of Medicine, Perelman School of Medicine, Professor of \\ Medicine, Vice Chair for Education and Inpatient Services, Philadelphia, USA.
}

J Gen Intern Med 27(3):270-2

DOI: $10.1007 / \mathrm{s} 11606-011-1942-\mathrm{Z}$

(C) Society of General Internal Medicine 2011

$\mathrm{H}$ andoffs in healthcare are inherently dangerous. Reviews of malpractice claims involving communication failures in a variety of settings demonstrate that 19 $43 \%$ are related to handoffs. ${ }^{1,2}$ In national patient safety culture surveys, healthcare providers consistently report that handoffs and transitions in their hospitals are hazardous with information frequently "falling between the cracks". 3 Although a number of regulatory and national patient safety organizations have called for a standardized approach to improve handoffs, there is a general consensus in the medical community that handoff errors continue to occur at unacceptable rates. ${ }^{4}$

Handoff systems have historically been unstructured in hospitals and handoff skills have not been taught in medical education. Resident handoffs have received considerable attention recently because their number has increased with each iteration of the Accreditation Council of Graduate Medical Education duty-hour standards. ${ }^{5}$ In 2008, the Institute of Medicine recommended ways to improve safety conditions and educational experiences during residency with the goal of embedding patient safety attitudes and behaviors into residents' future practice. ${ }^{6}$ Not surprisingly, handoff communication skills received heavy emphasis. Although several institutions have heeded these calls to action and made laudable efforts to improve resident handoffs through education and the creation of standardized communication tools, most residency programs still struggle with how to make resident handoffs safer. ${ }^{7}$

In this issue of JGIM, Helms and colleagues at the University of Virginia contribute in a significant way to the handoff education literature using a unique approach. ${ }^{8}$ They conducted a three-part study that assessed internal medicine resident attitudes about the handoff process, systematically analyzed consecutive handoffs to assess content and duration, and interviewed residents voted by their peers as

Published online December 22, 2011 "top performers" in handoffs in order to identify best practices. They had four main findings: 1) residents have different views about the handoff process and content; 2) up to $40 \%$ of residents did not expect to make decisions on their cross-cover patients; 3) consecutive or "double" handoffs were significantly shorter and associated with degradation of important clinical information; and 4) identifying and interviewing residents with excellent handoff skills was an effective way to identify best practices that could be used as the foundation for programmatic improvement. We will comment on each of these findings and discuss how they relate to the current state of handoffs within residency training.

The residents in this study agreed upon the ultimate purpose of handoffs, but they lacked a shared mental model of how handoffs should be performed. Seventy percent of residents believed handoffs should be problem-based while $30 \%$ believed that they should be system-based; $41 \%$ believed that handoffs should offer a chance to discuss alternative diagnoses and treatments, while $59 \%$ did not. Additional disparate views on the organization and content of handoffs were also reported. With such varied beliefs in how a handoff should be executed, it is not surprising that $76 \%$ of residents in this study reported finding major errors in handoffs "all or most of the time."

Forty percent of residents in this study believed that they should not be expected to make many decisions on crosscover patients. When one factors in nighttime coverage, weekend coverage, and other shift-based coverage models related to restricted resident work hours, patients in the hospital are covered by a "cross-cover" doctor approximately $50 \%$ of the time. Lack of patient ownership due to a shift-work mentality among residents concerns medical educators who strive to foster professionalism and enforce duty hours at the same time. As programs evolve and adjust to the new duty hour requirements with new cross-coverage and shift work models, the type of decisions made during cross-cover periods will also need to expand and change. For example, most cross-cover residents would accept and dutifully perform the task of following-up on a pending lab result overnight, but how many of them would respond to a nurses call to reassess the need for a foley catheter or agree to speak in person with a patient's family member who arrived late at night? Although one published handoff 
assessment tool includes professionalism as a component, the majority of the published handoff curricula in medicine have focused on describing and assessing the discrete safety behaviors to be used during a handoff. ${ }^{9}$ Going forward we will need to seek out ways to incorporate professionalism teaching and assessment into handoff curricula as we strive to foster resident ownership and patient-centered care at all hours of the day.

Consecutive or "double handoffs"were found in this study to be shorter and to result in degradation of information. This is not surprising given the natural tendency for information to be lost as it is transmitted through multiple providers. This degradation of information is compounded by "double handoffs" in which residents do not know the patient and are less likely to provide key clinical details accurately. Eliminating or minimizing double handoffs is the ideal solution but not always possible in this era of constrained duty hours. Program directors should seek innovative ways to involve the primary team in the handoff by including the attending, fellow, or nurse at the handoff or having the primary team sign out to the afternoon and the night team together.

The final lesson learned from Helm et al. is perhaps the most important. In this study, residents with excellent handoff skills were identified by their peers and used as the foundation for the redesign of handoff best practices through a strategy called appreciative inquiry. Appreciative inquiry is an approach to organizational change developed in the 1980s as a novel way to change culture. ${ }^{10}$ Organizations that use this approach begin by identifying and "appreciating," what is best about their system and using that as a starting point for improvement, as opposed to the more common approach of starting with the problem and solving it from there. Many residency program leaders likely use an appreciative inquiry approach in everyday practice without considering it a discrete strategy but could perhaps learn from these authors by expanding and formalizing its use in specific areas where improvement is needed. At the University of Virginia, the appreciative inquiry approach led to important process changes such as identifying aspects of their computerized handoff tool that were unhelpful and error-prone as well as proposing changes to the software system that would match their idealized verbal handoff design. We assert that compared with verbal handoff skills, written handoff skills will prove harder to improve and study in healthcare. Assigning accountability and providing feedback for the electronic documentation of physicians is challenging due to multiple authors, the evolving nature of the electronic medical record, and the problems associated with copy and paste. ${ }^{11}$

As handoff education evolves in residency training, it will be important to create a shared mental model with residents that can be articulated and practiced in individual programs. Creating a shared vision for how high risk processes and procedures such as handoffs should be performed in order to reduce harm and maintain safe outcomes is a key feature of other high-risk organizations, such as the airline industry. In a sense we are discovering in healthcare what other high risk industries have known for years - we will never eliminate handoffs, so our goal must be to become preoccupied with how and why they can fail. A common thread in the handoff research and innovation at the University of Virginia was the use of residents as the change agents of their own handoff systems. This exemplifies the "bottom-up" approach to engaging residents in institutional quality improvement and patient safety initiatives. ${ }^{11}$ This is in contrast to "topdown" approaches initiated by some institutions. While each approach has advantages and disadvantages, "bottom-up" approaches - when well supported and resourced-allow residents and programs to address problems that matter most to them and tailor their solutions to their local environment. Since most hospitals continue to experience handoff errors and most residency programs are just beginning to standardize and teach handoffs, we believe that efforts to improve handoff communications occupy the "sweet spot" and will remain an important patient safety target for residency programs and teaching hospitals for years to come.

Acknowledgement: This work was supported in part by the Josiah Macy Jr Foundation.

Corresponding Author: Lisa M. Bellini, MD; Department of Medicine, Perelman School of Medicine, Professor of Medicine, Vice Chair for Education and Inpatient Services, Philadelphia, USA (e-mail: Lisa.Bellini@uphs.upenn.edu).

\section{REFERENCES}

1. Singh H, Thomas EJ, Petersen LA, Studdert DM. Medical errors involving trainees: A study of closed malpractice claims from 5 insurers. Arch Int Med. 2007;167(19):2030-2036.

2. Greenberg CC, Regenbogen SE, Studdert DM, et al. Patterns of communication breakdowns resulting in injury to surgical patients. J Am Coll Surg. 2007;204:533-540.

3. Agency for Healthcare Quality and Research. Hospital Survey on Patient Safety Culture: 2010 User Comparative Database Report. http://www. ahrq.gov/qual/hospsurvey 10/hosp10tabc8.htm. Accessed Oct 27, 2011

4. Arora V, Johnson J. National patient safety goals. A model for building a standardized hand-off protocol. Jt Comm J Qual Patient Saf. 006;32:646-655.

5. American College of Graduate Medical Education Standards. Common Program Requirements. http://www.acgme.org/acWebsite/home/ Common_Program_Requirements_07012011.pdf. Accessed October 28,2011

6. Resident Duty Hours: Enhancing Sleep, Supervision, and Safety. Ulmer C, Wolman DM, Johns MME, eds. Committee on Optimizing Graduate Medical Trainee (Resident) Hours and Work Schedule to Improve Patient 
Safety, Institute of Medicine. Washington, DC: The National Academies Press; 2008

7. Riesenberg L, Leitzsch J, Massucci JL, et al. Residents and attending physicians' handoffs: a systematic review of the literature. Acad Med. 2009;84:1775-1787.

8. Helms AS, Perez TE, Baltz J, Donowitz G, Hoke G, Bass EJ, Plews-Ogan ML. Use of an Appreciative Inquiry Approach to Improve Resident SignOut in an Era of Multiple Shift Changes.J Gen Intern Med. 2011; doi: 10.1007/s11606-011-1885-4
9. Farnan JM, Paro JAM, Rodriguez RM, et al. Hand-off education and evaluation: Piloting the observed simulated hand-off experience (OSHE). J Gen Intern Med. 2010;25(2):129-134.

10. Cooperrider DL, Srivastva S. Appreciative inquiry in organizational life. In R. Woodman \& W. Pasmore (eds.) Research in Organizational Change and Development: Volume 1 (pp. 129-169). Greenwich, CT: JAI Press; 1987

11. Siegler EL, Adelman R. Copy and paste: a remediable hazard of electronic health records. Am J Med. 2009;122(6):495-496. 